\title{
ASSOCIAÇÃO ENTRE TROPISMO FACETÁRIO E DOENÇA DEGENERATIVA DE DISCO LOMBAR
}

\author{
ASSOCIATION BETWEEN FACET TROPISM AND LUMBAR DEGENERATIVE DISC DISEASE \\ ASOCIACIÓN ENTRE TROPISMO FACETARIO Y ENFERMEDAD DEGENERATIVA \\ DEL DISCO LUMBAR
}

Alexandre Barros Costa ${ }^{1}$, Thiago Mattar ${ }^{1}$, Paulo Roberto Appolonio ${ }^{1}$, Calo Yoshino ${ }^{1}$, Adriano Massayuki Yonezaki ${ }^{1}$, LuCiano Miller Reis Rodrigues ${ }^{1}$

\begin{abstract}
RESUMO
Objetivo: Documentar a presença de degeneração de disco e tropismo facetário em pacientes portadores de dor lombar crônica e sua distribuição por sexo e faixa etária. Avaliar também a associação de tropismo facetário e degeneração discal lombar além de avaliar a orientação das facetas de acordo com sexo e faixa etária. Métodos: Estudo retrospectivo de imagens de ressonância magnética obtidas em 288 pacientes ( $\mathrm{N}=$ 288; 118 homens e 170 mulheres) com média de idade de 53,33 anos, portadores de dor lombar crônica. As imagens foram avaliadas por dois médicos assistentes especialistas em cirurgia da coluna para avaliar e quantificar a orientação das facetas, o tropismo facetário e o grau de degeneração discal dos níveis L3-L4, L4-L5 e L5-S1. Foi analisada a associação entre tropismo facetário e doença degenerativa discal, além de associação com sexo e idade. Resultados: Observa-se que 85,8\% dos discos apresentam classificação de Pfirrmann superior ou igual ao Tipo III. Com relação ao grau de degeneração discal, não houve diferença entre os sexos e aumentou com o aumento da faixa etária. Com relação ao grau de tropismo, não difere entre os níveis avaliados e o sexo, aumenta de acordo com a elevação da faixa etária. Houve aumento do grau do degeneração discal com o aumento do grau de tropismo facetário. Conclusão: A maioria dos discos intervertebrais analisados de pacientes com dor lombar crônica encontram-se degenerados e grau de degeneração aumenta com a idade. O grau de tropismo facetário aumenta com a idade e se relaciona com o grau de degeneração discal.
\end{abstract}

Descritores: Coluna vertebral; Disco intervertebral; Dor lombar; Tropismo; Estudos retrospectivos.

\begin{abstract}
Objective: To document the presence of disc degeneration and facet tropism in patients with chronic low back pain and its distribution by sex and age. We also evaluated the association between facet tropism and lumbar disc degeneration and the orientation of the facets according to sex and age. Methods: Retrospective study of MRls obtained from 288 patients ( $N=288$, 118 men and 170 women) with mean age of 53.33 years, and chronic low back pain. The images were evaluated by two physicians specialized in surgery of the spine to assess and quantify the orientation of the facets, facet tropism and degree of disc degeneration at L3-L4, L4-L5 and L5-S1. We analyzed the association between facet tropism and degenerative disc disease, and association with sex and age. Results: It is observed that $85.8 \%$ of the disks have Pfirrmann rating greater or equal to Type III. Concerning the degree of disc degeneration, there was no difference between sexes and increased with increasing age. There was an increase in the degree of disc degeneration with increasing degree of facet tropism. Conclusion: Most of the intervertebral discs analyzed in patients with chronic low back pain are degenerate and the degree of degeneration increases with age. The degree of facet tropism increases with age and is related to the degree of disc degeneration.
\end{abstract}

Keywords: Spine; Intervertebral disc; Low back pain; Tropism; Retrospective studies.

RESUMEN

Objetivo: documentar la presencia de degeneración de disco y tropismo facetario en pacientes con dolor crónico de en la columna lumbar y su distribución por sexo y edad. También evaluamos la asociación del tropismo facetario y la degeneración del disco lumbar, además de la orientación de las facetas de acuerdo con sexo y edad. Métodos: Estudio retrospectivo de imágenes de resonancia magnética obtenidas en 288 pacientes ( $N=288$; 118 hombres y 170 mujeres) con edad media de 53,33 años y dolor crónico en la región lumbar de columna. Las imágenes fueron evaluadas por dos médicos especialistas en cirugía de la columna vertebral para valorar y cuantificar la orientación de las facetas, el tropismo facetario y el grado de degeneración de disco en L3-L4, L4-L5 y L5-S1. Se analizó la asociación entre tropismo facetario y la enfermedad degenerativa del disco y la asociación con el sexo y la edad. Resultados: Se observa que 85,8\% de los discos tienen clasificación de Pfirrmann mayor o igual que Tipo III. En cuanto al grado de degeneración del disco, no fue diferente entre sexos y aumentó con la edad. En cuanto al grado de tropismo, no hay diferencia entre los niveles evaluados y el sexo y aumenta con la edad. Hubo un aumento en el grado de degeneración de disco con el aumento del grado del tropismo facetario. Conclusión: La mayoría de los discos intervertebrales analizados en los pacientes con dolor lumbar crónico se degeneran y el grado de degeneración aumenta con la edad. El grado de tropismo facetario aumenta con la edad y se relaciona con el grado de degeneración del disco.

Descriptores: Columna vertebral; Disco intervertebral; Dolor de la región lumbar; Tropismo; Estudios Retrospectivos.

Departamento de Ortopedia eTraumatologia da Faculdade de Medicina do ABC - Santo André, SP, Brasil.

Trabalho realizado no Grupo de Cirurgia da Coluna Vertebral da Faculdade de Medicina do ABC - Santo André, SP, Brasil.

Correspondência: RuaTucuma, 199, apto 141.São Paulo, SP. Brasil 01455- 010. luciano.miller@uol.com.br

Coluna/Columna. 2013; 12(2): 133-7 


\section{INTRODUÇÃO}

A dor lombar (lombalgia) é um importante problema de saúde e com grande impacto econômico nos países industrializados, sendo uma das principais causas de despesas médicas, absenteísmo e incapacidade para o trabalho. ${ }^{1}$ Relatórios recentes indicam que a dor lombar pode ser causada por alterações em estruturas específicas da coluna, incluindo anormalidades na articulação interapofisária, patologia discal e artropatia da articulação sacroilíaca. ${ }^{2-5}$

A orientação facetaria é o ângulo da articulação da faceta no plano transversal. Tropismo facetário é definido como assimetria entre os ângulos facetários à esquerda e à direita, tendo um deles uma orientação mais sagital em relação ao contralateral. ${ }^{6} \mathrm{~A}$ orientação da faceta e tropismo facetário podem ter relação com as alterações degenerativas da coluna, quer como causa ou com consequência de forças anormais produzidas pela degeneração. ${ }^{7}$

Apesar dos estudos extensivos o significado clínico do tropismo e da orientação das facetas ainda não está bem estabelecido. Cassidy et al. ${ }^{8}$ e Vanharanta et al. ${ }^{9}$ não encontram relação entre tropismo facetário e degeneração discal lombar. No entanto, Noren et al. ${ }^{10}$ relataram que o tropismo facetário está associado ao desenvolvimento de hérnia e degeneração discal em todos os níveis lombares.

Tanto a degeneração discal quanto a artrose facetaria têm relação direta com a faixa etária do paciente, iniciando-se em torno dos 30 anos de idade e quase sempre presente após os 60 anos. ${ }^{6}$ Wang et al. ${ }^{11}$ observaram que a orientação da facetas L4-L5 diminuem seu ângulo no plano sagital com o envelhecimento. Estudos mostraram que a orientação coronal das facetas é pouco resistente as forças de cisalhamento, tendendo a girar no lado onde a faceta é mais coronalmente orientada, o que pode levar a instabilidade. ${ }^{12}$

Os objetivos do estudo foram: documentar a presença de degeneração discal e tropismo facetário em pacientes portadores de dor lombar crônica e sua distribuição por sexo e faixa etária. Também avaliar a associação de tropismo facetário e degeneração discal lombar além de avaliar a orientação das facetas de acordo com sexo e faixa etária.

\section{MATERIAIS E MÉTODOS}

Foram avaliadas as ressonância magnéticas de 288 pacientes portadores de dor lombar crônica atendidos no Ambulatório de Cirurgia da Coluna no período de janeiro a dezembro de 2011. Critérios de inclusão: presença de dor lombar por um período superior a três meses. Critérios de exclusão: presença de cirurgia prévia, fratura, tumor ou infecção na coluna, além de deformidades como escoliose, cifose ou má formações congênitas.

Tropismo facetário: dois assistentes do Grupo de Coluna foram responsáveis pela medição dos ângulos das facetas articulares dos níveis L3-L4, L4-L5, L5-S1, totalizando 864 níveis analisados. Os ângulos das facetas articulares foram medidos através de cortes axiais na sequência $T 1$ da ressonância magnética de 1,5 Tesla, as linhas de referência foram: aspecto posterior do corpo vertebral (perpendicular ao plano sagital) e uma linha traçada tangenciando a faceta superior do processo articular. (Figura 1) O grau de tropismo (assimetria) foi classificado de acordo com o método descrito por Vanharata et al. ${ }^{9}$ que definiu tropismo ausente para diferenças menores de $7^{\circ}$, moderado com diferença entre $7^{\circ}$ e $15^{\circ}$, e tropismo grave com mais de $15^{\circ}$.

A classificação da degeneração discal foi feita segundo os critérios de Pfirrmann, ${ }^{13}$ pelos mesmos avaliadores do tropismo facetário, foram avaliados os níveis L3-L4, L4-L5, L5-S1, totalizando 864 níveis analisados.

O grau de degeneração discal segundo Pfirrmann ${ }^{13}$ e o grau de tropismo facetário foram descritas segundo sexo e comparadas entre os sexos com uso de testes Mann-Whitney (Kirkwood e Sterne). Os dados foram descritas por faixas etárias e comparadas utilizando testes Kruskal-Wallis (Kirkwood e Sterne). O grau do Pfirrmann nos níveis analisados foi comparado em relação ao grau de tropismo facetário e verificada a existência de relação entre as duas escalas em nível com uso de correlações de Spearman (Kirkwood e Sterne).

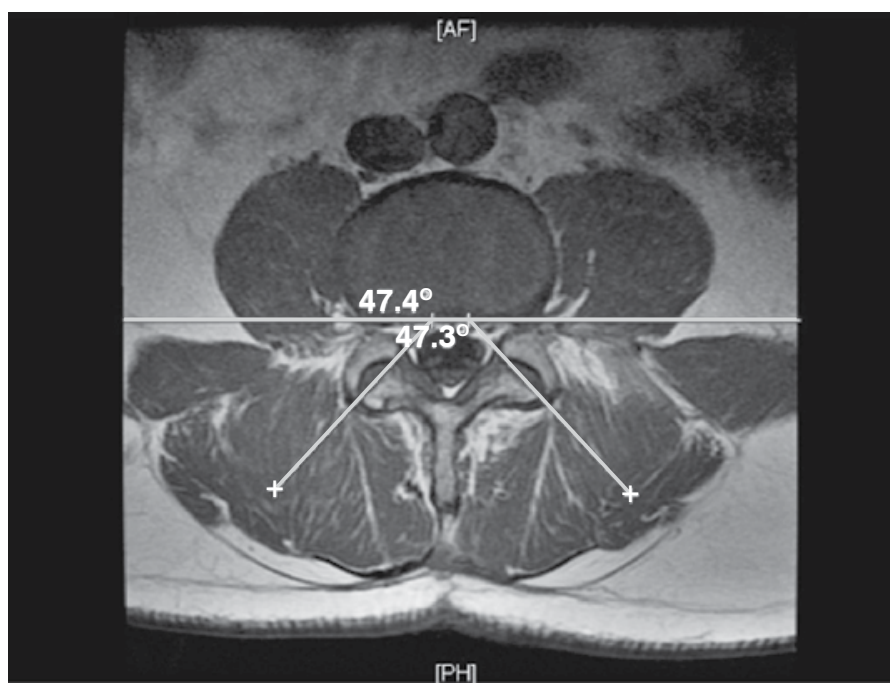

Figura 1. Medição do tropismo facetário.

O ângulo médio da orientação das facetas por nível descrito segundo sexo e faixa etária com uso de médias e desvio padrão e comparados entre as categorias de sexo e faixa etária com uso de análise de variâncias (ANOVA) com dois fatores.

Os testes foram realizados com nível de significância de 5\%.

\section{RESULTADOS}

Foram analisados 288 pacientes, sendo 170 do sexo feminino (59\%) e 118 do sexo masculino (41\%), com média de idade 53,33 anos (desvio padrão $\pm 12,84$ ). A maioria se encontrava na faixa etária de 40 a 60 anos (54\%). (Tabela 1)

Com relação à avaliação da degeneração discal segundo Pfirmann ${ }^{13}$, o tipo III foi o mais frequente nos níveis $L 3-L 4$ e $L 5$ S1, já no nível L4-L5 o tipo IV foi o mais prevalente. Observa-se que $85,8 \%$ dos discos apresentam Pfirmann ${ }^{13}$ superior ou igual ao Tipo III. (Tabela 2)

A distribuição do Pfirrmann ${ }^{13}$ que acordo com o sexo mostrou que as mulheres apresentam grau de degeneração estatisticamente maior no nível L3-L4 ( $p=0,002)$, em relação aos demais níveis e ao total de níveis analisados não houve diferença entre os sexos. (Tabela 3)

Na Tabela 4, observa-se que grau de degeneração discal, segundo o Pfirrmann ${ }^{13}$, foi estatisticamente maior com aumento da faixa etária nos níveis $L 3-L 4$ e L4-L5 ( $p<0,01)$ e em relação ao total de níveis analisados $(p<0,05)$. Quanto ao nível L5S1, observa-se uma tendência a aumento desta relação, porém o grau de degeneração é estatisticamente semelhante nas três faixas etárias analisadas $(p=0,253)$.

A avaliação do grau de Tropismo Facetário mostrou que o grau de assimetrias são bastante semelhantes entre os níveis estudados. (Tabela 5)

Tabela 1. Distribuição dos pacientes segundo idade, média de idade e faixa etária.

\begin{tabular}{c|c|c}
\hline Variável & N & $\%$ \\
\hline Sexo & & \\
\hline Feminino & 170 & 59,0 \\
\hline Masculino & 118 & 41,0 \\
\hline Faixa etária & & \\
\hline$<40$ anos & 52 & 18,1 \\
\hline 40 a 60 anos & 156 & 54,2 \\
\hline$>60$ anos & 80 & 27,8 \\
\hline Total & 288 & 100 \\
\hline
\end{tabular}


O resultado do grau do tropismo facetário segundo o sexo mostrou que o grau de assimetria é estatisticamente maior nos homens no nível L5-S1 $(p=0,027)$. (Tabela 6)

$\mathrm{Na}$ Tabela 7, observa-se que a severidade do Tropismo Facetário aumenta estatisticamente com o aumento da faixa etária nos níveis L3-L4, L4-L5 e na soma dos níveis avaliados $(p<0,05)$.

$\mathrm{Na}$ Tabela 8, observa-se quanto maior o grau de degeneração, segundo Pfirrmann ${ }^{13}$, maior o grau de severidade do tropismo facetário e vice-versa $(p<0,05)$.

$\mathrm{Na}$ avaliação do grau de orientação das facetas (ângulo médio por nível) por sexo e faixa etária não houve diferença estatística $(p>0,05)$. (Tabela 9)

Tabela 2. Distribuição da amostra segundo o Pfirrmann. ${ }^{13}$

\begin{tabular}{c|c|c|c|c|c|c|c|c}
\hline \multirow{2}{*}{ Pfirrmann } & \multicolumn{2}{|c|}{ L3-L4 } & \multicolumn{2}{c|}{ L4-L5 } & \multicolumn{2}{c|}{ L5-S1 } & \multicolumn{2}{c}{ Todos os discos } \\
\cline { 2 - 9 } & $\mathbf{N}$ & $\%$ & $\mathbf{N}$ & $\%$ & $\mathbf{N}$ & $\%$ & $\mathbf{N}$ & $\%$ \\
\hline Tipo I & 0 & 0,0 & 5 & 1,7 & 0 & 0,0 & 5 & 0,6 \\
\hline Tipo II & 51 & 17,7 & 34 & 11,8 & 33 & 11,5 & 118 & 13,7 \\
\hline Tipo III & 120 & 41,7 & 88 & 30,6 & 102 & 35,4 & 310 & 35,9 \\
\hline Tipo IV & 87 & 30,2 & 99 & 34,4 & 93 & 32,3 & 279 & 32,3 \\
\hline Tipo V & 30 & 10,4 & 62 & 21,5 & 60 & 20,8 & 152 & 17,6 \\
\hline Total & 288 & 100 & 288 & 100 & 288 & 100 & 864 & 100 \\
\hline
\end{tabular}

Tabela 3. Distribuição do Pfirrmann ${ }^{13}$ segundo o sexo.

\begin{tabular}{|c|c|c|c|c|c|c|c|}
\hline \multirow{3}{*}{ Variável } & \multicolumn{4}{|c|}{ Sexo } & & & \multirow{3}{*}{$\mathbf{p}$} \\
\hline & \multicolumn{2}{|c|}{ Feminino } & \multicolumn{2}{|c|}{ Masculino } & \multicolumn{2}{|c|}{ Total } & \\
\hline & $\mathbf{N}$ & $\%$ & $\mathbf{N}$ & $\%$ & $\mathbf{N}$ & $\%$ & \\
\hline Pfirrmann ${ }^{13}$ L3-L4 & & & & & & & 0,002 \\
\hline Tipo ॥ & 29 & 17,1 & 22 & 18,6 & 51 & 17,7 & \\
\hline Tipo III & 58 & 34,1 & 62 & 52,5 & 120 & 41,7 & \\
\hline Tipo IV & 57 & 33,5 & 30 & 25,4 & 87 & 30,2 & \\
\hline Tipo V & 26 & 15,3 & 4 & 3,4 & 30 & 10,4 & \\
\hline Pfirrmann L4-L5 & & & & & & & 0,201 \\
\hline Tipo I & 5 & 2,9 & 0 & & 5 & 1,7 & \\
\hline Tipo ॥ & 14 & 8,2 & 20 & 16,9 & 34 & 11,8 & \\
\hline Tipo III & 58 & 34,1 & 30 & 25,4 & 88 & 30,6 & \\
\hline Tipo IV & 46 & 27,1 & 53 & 44,9 & 99 & 34,4 & \\
\hline Tipo V & 47 & 27,6 & 15 & 12,7 & 62 & 21,5 & \\
\hline Pfirrmann L5-S1 & & & & & & & 0,912 \\
\hline Tipo ॥ & 15 & 8,8 & 18 & 15,3 & 33 & 11,5 & \\
\hline Tipo III & 69 & 40,6 & 33 & 28,0 & 102 & 35,4 & \\
\hline Tipo IV & 50 & 29,4 & 43 & 36,4 & 93 & 32,3 & \\
\hline Tipo V & 36 & 21,2 & 24 & 20,3 & 60 & 20,8 & \\
\hline $\begin{array}{c}\text { Pfirrmann } \\
\text { (maior grau) }\end{array}$ & & & & & & & 0,749 \\
\hline Tipo II & 7 & 4,1 & 4 & 3,4 & 11 & 3,8 & \\
\hline Tipo III & 31 & 18,2 & 16 & 13,6 & 47 & 16,3 & \\
\hline Tipo IV & 67 & 39,4 & 61 & 51,7 & 128 & 44,4 & \\
\hline Tipo V & 65 & 38,2 & 37 & 31,4 & 102 & 35,4 & \\
\hline Total & 170 & 100 & 118 & 100 & 288 & 100 & \\
\hline
\end{tabular}

Resultado do teste Mann-Whitney.
Tabela 4. Distribuição do Pfirrmann ${ }^{13}$ segundo as faixas etárias

\begin{tabular}{|c|c|c|c|c|c|c|c|c|c|}
\hline \multirow{3}{*}{ Variável } & \multicolumn{6}{|c|}{ Faixa etária } & & & \multirow{3}{*}{$\mathbf{p}$} \\
\hline & \multicolumn{2}{|c|}{$<40$ anos } & \multicolumn{2}{|c|}{40 a 60 anos } & \multicolumn{2}{|c|}{$>60$ anos } & \multicolumn{2}{|c|}{ Total } & \\
\hline & $\mathbf{N}$ & $\%$ & $\mathbf{N}$ & $\%$ & $\mathbf{N}$ & $\%$ & $\mathbf{N}$ & $\%$ & \\
\hline Pfirrmann $^{13}$ L3-L4 & & & & & & & & & $<0,001$ \\
\hline Tipo II & 25 & 48,1 & 24 & 15,4 & 2 & 2,5 & 51 & 17,7 & \\
\hline Tipo III & 21 & 40,4 & 66 & 42,3 & 33 & 41,3 & 120 & 41,7 & \\
\hline Tipo IV & 4 & 7,7 & 46 & 29,5 & 37 & 46,3 & 87 & 30,2 & \\
\hline Tipo V & 2 & 3,8 & 20 & 12,8 & 8 & 10,0 & 30 & 10,4 & \\
\hline Pfirrmann L4-L5 & & & & & & & & & $<0,001$ \\
\hline Tipo I & 3 & 5,8 & 0 & 0,0 & 2 & 2,5 & 5 & 1,7 & \\
\hline Tipo II & 16 & 30,8 & 16 & 10,3 & 2 & 2,5 & 34 & 11,8 & \\
\hline Tipo III & 22 & 42,3 & 42 & 26,9 & 24 & 30,0 & 88 & 30,6 & \\
\hline Tipo IV & 11 & 21,2 & 62 & 39,7 & 26 & 32,5 & 99 & 34,4 & \\
\hline Tipo V & 0 & 0,0 & 36 & 23,1 & 26 & 32,5 & 62 & 21,5 & \\
\hline Pfirrmann L5-S1 & & & & & & & & & 0,253 \\
\hline Tipo II & 9 & 17,3 & 20 & 12,8 & 4 & 5,0 & 33 & 11,5 & \\
\hline Tipo III & 16 & 30,8 & 52 & 33,3 & 34 & 42,5 & 102 & 35,4 & \\
\hline Tipo IV & 21 & 40,4 & 54 & 34,6 & 18 & 22,5 & 93 & 32,3 & \\
\hline Tipo V & 6 & 11,5 & 30 & 19,2 & 24 & 30,0 & 60 & 20,8 & \\
\hline $\begin{array}{c}\text { Pfirrmann } \\
\text { (maior grau) }\end{array}$ & & & & & & & & & $<0,00$ \\
\hline Tipo II & 7 & 13,5 & 4 & 2,6 & 0 & 0,0 & 11 & 3,8 & \\
\hline Tipo III & 13 & 25,0 & 22 & 14,1 & 12 & 15,0 & 47 & 16,3 & \\
\hline Tipo IV & 24 & 46,2 & 74 & 47,4 & 30 & 37,5 & 128 & 44,4 & \\
\hline Tipo V & 8 & 15,4 & 56 & 35,9 & 38 & 47,5 & 102 & 35,4 & \\
\hline Total & 52 & 100 & 156 & 100 & 80 & 100 & 288 & 100 & \\
\hline
\end{tabular}

Tabela 5. Distribuição da amostra segundo o tropismo facetário.

\begin{tabular}{c|c|c|c|c|c|c|c|c}
\hline \multirow{2}{*}{ Tropismo } & \multicolumn{2}{|c|}{ L3-4 } & \multicolumn{2}{c|}{ L4-L5 } & \multicolumn{2}{c|}{ L5-S1 } & \multicolumn{2}{c}{ Todos os discos } \\
\cline { 2 - 9 } & $\mathbf{N}$ & $\mathbf{\%}$ & $\mathbf{N}$ & $\mathbf{\%}$ & $\mathbf{N}$ & $\mathbf{\%}$ & $\mathbf{N}$ & $\%$ \\
\hline Ausente & 171 & 59,4 & 152 & 52,8 & 144 & 50,0 & 467 & 54,1 \\
\hline Moderado & 90 & 31,3 & 104 & 36,1 & 104 & 36,1 & 298 & 34,5 \\
\hline Severo & 27 & 9,4 & 32 & 11,1 & 40 & 13,9 & 99 & 11,5 \\
\hline Total & 288 & 100 & 288 & 100 & 288 & 100 & 864 & 100 \\
\hline
\end{tabular}

Tabela 6. Distribuição de tropismo facetário segundo sexo.

\begin{tabular}{|c|c|c|c|c|c|c|c|}
\hline \multirow{3}{*}{ Variável } & \multicolumn{4}{|c|}{ Sexo } & & & \multirow{3}{*}{$\mathbf{p}$} \\
\hline & \multicolumn{2}{|c|}{ Feminino } & \multicolumn{2}{|c|}{ Masculino } & \multicolumn{2}{|c|}{ Total } & \\
\hline & $\mathbf{N}$ & $\%$ & $\mathbf{N}$ & $\%$ & $\mathbf{N}$ & $\%$ & \\
\hline Tropismo L3-L4 & & & & & & & 0,490 \\
\hline Ausente & 100 & 58,8 & 71 & 60,2 & 171 & 59,4 & \\
\hline Moderado & 49 & 28,8 & 41 & 34,7 & 90 & 31,3 & \\
\hline Severo & 21 & 12,4 & 6 & 5,1 & 27 & 9,4 & \\
\hline Tropismo L4-L5 & & & & & & & 0,893 \\
\hline Ausente & 87 & 51,2 & 65 & 55,1 & 152 & 52,8 & \\
\hline Moderado & 68 & 40,0 & 36 & 30,5 & 104 & 36,1 & \\
\hline Severo & 15 & 8,8 & 17 & 14,4 & 32 & 11,1 & \\
\hline Tropismo L5-S1 & & & & & & & 0,027 \\
\hline Ausente & 93 & 54,7 & 51 & 43,2 & 144 & 50,0 & \\
\hline Moderado & 59 & 34,7 & 45 & 38,1 & 104 & 36,1 & \\
\hline Severo & 18 & 10,6 & 22 & 18,6 & 40 & 13,9 & \\
\hline Tropismo (maior grau) & & & & & & & 0,856 \\
\hline Ausente & 32 & 18,8 & 26 & 22,0 & 58 & 20,1 & \\
\hline Moderado & 92 & 54,1 & 55 & 46,6 & 147 & 51,0 & \\
\hline Severo & 46 & 27,1 & 37 & 31,4 & 83 & 28,8 & \\
\hline Total & 170 & 100 & 118 & 100 & 288 & 100 & \\
\hline
\end{tabular}


Tabela 7. Distribuição do grau de tropismo facetário por faixa etária.

\begin{tabular}{|c|c|c|c|c|c|c|c|c|c|}
\hline \multirow{3}{*}{ Variável } & \multicolumn{6}{|c|}{ Faixa etária } & & & \\
\hline & \multicolumn{2}{|c|}{$<40$ anos } & \multicolumn{2}{|c|}{40 a 60 anos } & \multicolumn{2}{|c|}{$>60$ anos } & \multicolumn{2}{|c|}{ Total } & p \\
\hline & $\mathbf{N}$ & $\%$ & $\mathbf{N}$ & $\%$ & $N$ & $\%$ & $\mathbf{N}$ & $\%$ & \\
\hline Tropismo L3-L4 & & & & & & & & & 0,034 \\
\hline Ausente & 39 & 75,0 & 87 & 55,8 & 45 & 56,3 & 171 & 59,4 & \\
\hline Moderado & 11 & 21,2 & 55 & 35,3 & 24 & 30,0 & 90 & 31,3 & \\
\hline Severo & 2 & 3,8 & 14 & 9,0 & 11 & 13,8 & 27 & 9,4 & \\
\hline Tropismo L4-L5 & & & & & & & & & 0,006 \\
\hline Ausente & 37 & 71,2 & 78 & 50,0 & 37 & 46,3 & 152 & 52,8 & \\
\hline Moderado & 13 & 25,0 & 63 & 40,4 & 28 & 35,0 & 104 & 36,1 & \\
\hline Severo & 2 & 3,8 & 15 & 9,6 & 15 & 18,8 & 32 & 11,1 & \\
\hline Tropismo L5-S1 & & & & & & & & & 0,206 \\
\hline Ausente & 30 & 57,7 & 75 & 48,1 & 39 & 48,8 & 144 & 50,0 & \\
\hline Moderado & 20 & 38,5 & 55 & 35,3 & 29 & 36,3 & 104 & 36,1 & \\
\hline Severo & 2 & 3,8 & 26 & 16,7 & 12 & 15,0 & 40 & 13,9 & \\
\hline $\begin{array}{c}\text { Tropismo } \\
\text { (maior grau) }\end{array}$ & & & & & & & & & 0,002 \\
\hline Ausente & 16 & 30,8 & 28 & 17,9 & 14 & 17,5 & 58 & 20,1 & \\
\hline Moderado & 30 & 57,7 & 83 & 53,2 & 34 & 42,5 & 147 & 51,0 & \\
\hline Severo & 6 & 11,5 & 45 & 28,8 & 32 & 40,0 & 83 & 28,8 & \\
\hline Total & 52 & 100 & 156 & 100 & 80 & 100 & 288 & 100 & \\
\hline
\end{tabular}

Resultado do teste Kruskal-Wallis

Tabela 8. Relação entre Pfirrmann ${ }^{13}$ e tropismo facetário.

\begin{tabular}{|c|c|c|c|c|c|c|c|c|c|c|}
\hline \multirow{3}{*}{ Disco } & \multirow{3}{*}{ Pfirrmann } & \multicolumn{6}{|c|}{ Tropismo } & & & \multirow{3}{*}{ p } \\
\hline & & \multicolumn{2}{|c|}{ Ausente } & \multicolumn{2}{|c|}{ Moderado } & \multicolumn{2}{|c|}{ Severo } & \multicolumn{2}{|c|}{ Total } & \\
\hline & & $\mathbf{N}$ & $\%$ & $\mathbf{N}$ & $\%$ & $\mathbf{N}$ & $\%$ & $\mathbf{N}$ & $\%$ & \\
\hline \multirow{5}{*}{ L3-L4 } & Tipo II & 41 & 24,0 & 8 & 8,9 & 2 & 7,4 & 51 & 17,7 & \multirow{4}{*}{$<0,001$} \\
\hline & Tipo III & 75 & 43,9 & 39 & 43,3 & 6 & 22,2 & 120 & 41,7 & \\
\hline & Tipo IV & 47 & 27,5 & 29 & 32,2 & 11 & 40,7 & 87 & 30,2 & \\
\hline & Tipo V & 8 & 4,7 & 14 & 15,6 & 8 & 29,6 & 30 & 10,4 & \\
\hline & Total & 171 & 100 & 90 & 100 & 27 & 100 & 288 & 100 & \\
\hline \multirow{6}{*}{ L4-L5 } & Tipo I & 5 & 3,3 & 0 & 0,0 & 0 & 0,0 & 5 & 1,7 & \multirow{5}{*}{$<0,001$} \\
\hline & Tipo II & 30 & 19,7 & 4 & 3,8 & 0 & 0,0 & 34 & 11,8 & \\
\hline & Tipo III & 48 & 31,6 & 30 & 28,8 & 10 & 31,3 & 88 & 30,6 & \\
\hline & Tipo IV & 52 & 34,2 & 41 & 39,4 & 6 & 18,8 & 99 & 34,4 & \\
\hline & Tipo V & 17 & 11,2 & 29 & 27,9 & 16 & 50,0 & 62 & 21,5 & \\
\hline & Total & 152 & 100 & 104 & 100 & 32 & 100 & 288 & 100 & \\
\hline \multirow{5}{*}{ L5-S1 } & Tipo II & 27 & 18,8 & 0 & 0,0 & 6 & 15,0 & 33 & 11,5 & \multirow{4}{*}{$<0,001$} \\
\hline & Tipo III & 53 & 36,8 & 39 & 37,5 & 10 & 25,0 & 102 & 35,4 & \\
\hline & Tipo IV & 38 & 26,4 & 39 & 37,5 & 16 & 40,0 & 93 & 32,3 & \\
\hline & Tipo V & 26 & 18,1 & 26 & 25,0 & 8 & 20,0 & 60 & 20,8 & \\
\hline & Total & 144 & 100 & 104 & 100 & 40 & 100 & 288 & 100 & \\
\hline \multirow{5}{*}{$\begin{array}{c}\text { Mais } \\
\text { degenerado }\end{array}$} & Tipo II & 7 & 12,1 & 2 & 1,4 & 2 & 2,4 & 11 & 3,8 & \multirow{4}{*}{$<0,001$} \\
\hline & Tipo III & 15 & 25,9 & 26 & 17,7 & 6 & 7,2 & 47 & 16,3 & \\
\hline & Tipo IV & 18 & 31,0 & 74 & 50,3 & 36 & 43,4 & 128 & 44,4 & \\
\hline & Tipo V & 18 & 31,0 & 45 & 30,6 & 39 & 47,0 & 102 & 35,4 & \\
\hline & Total & 58 & 100 & 147 & 100 & 83 & 100 & 288 & 100 & \\
\hline
\end{tabular}

Tabela 9. Relação entre média da orientação das facetas por sexo e faixa etária.

\begin{tabular}{|c|c|c|c|c|c|c|c|c|}
\hline \multirow{2}{*}{ Variável } & \multirow{2}{*}{ Faixa etária } & \multicolumn{3}{|c|}{ Femnino } & \multicolumn{3}{|c|}{ Masculino } & \multirow{2}{*}{$\mathbf{p}^{*}$} \\
\hline & & Média & DP & $\mathbf{N}$ & Média & DP & $\mathbf{N}$ & \\
\hline \multirow{3}{*}{$\begin{array}{l}\text { Ângulo } \\
\text { L3-L4 }\end{array}$} & $<40$ anos & 51,11 & 6,83 & 28 & 54,56 & 11,04 & 24 & \multirow{3}{*}{0,457} \\
\hline & 40 a 60 anos & 54,98 & 10,25 & 87 & 54,54 & 10,78 & 69 & \\
\hline & $>60$ anos & 54,41 & 9,61 & 55 & 55,31 & 11,08 & 25 & \\
\hline p\# & & \multicolumn{6}{|c|}{0,337} & \\
\hline \multirow{3}{*}{$\begin{array}{c}\text { Ângulo } \\
\text { L4-L5 }\end{array}$} & $<40$ anos & 42,73 & 8,48 & 28 & 41,77 & 14,40 & 24 & \multirow{3}{*}{0,261} \\
\hline & 40 a 60 anos & 45,92 & 10,34 & 87 & 43,60 & 11,09 & 69 & \\
\hline & $>60$ anos & 43,34 & 11,41 & 55 & 47,50 & 11,45 & 25 & \\
\hline p\# & & \multicolumn{6}{|c|}{0,842} & \\
\hline \multirow{3}{*}{$\begin{array}{l}\text { Ângulo } \\
\text { L5-S1 }\end{array}$} & $<40$ anos & 41,50 & 7,86 & 28 & 39,64 & 9,18 & 24 & \multirow{3}{*}{0,303} \\
\hline & 40 a 60 anos & 42,74 & 10,81 & 87 & 41,12 & 9,09 & 69 & \\
\hline & $>60$ anos & 43,98 & 15,31 & 55 & 43,39 & 11,23 & 25 & \\
\hline p\# & & \multicolumn{6}{|c|}{0,365} & \\
\hline
\end{tabular}

Análise de variâncias (ANOVA). ${ }^{*}$ Resultado da comparação entre as faixas etárias. \# Resultado da comparação entre sexos.

\section{DISCUSSÃO}

A discopatia degenerativa é fenômeno bastante prevalente na população adulta, sendo uma das fontes de origem da lombalgia, segundo a literatura $40 \%$ das dores lombares crônicas são de origem discogênica. ${ }^{14} \mathrm{O}$ estudo desta alteração é de fundamental importância para melhor compreensão desta etiologia e visa por um tratamento mais específico para esses sintomas.

Nosso estudo avaliou pacientes com média de idade de 53,33 anos sendo o Tipo III seguido pelo IV de Pfirrmann os mais frequentes. Enquanto Lam et al. ${ }^{15}$ observaram a maior frequência dos tipos III seguidos II, no entanto, analisou uma população com média de idade de 38,9 anos, provavelmente o fator idade resultou na frequência menor de formas mais graves de degeneração discal. Dados da literatura confirmam que existe uma relação direta entre idade e o grau de degeneração discal na coluna lombar. ${ }^{16,17}$ Nossa análise observou o aumento da degeneração discal com o aumento da faixa etária, quanto à avaliação por nível mostrou aumento em L3-4 e L4-L5.

Segundo Zue ${ }^{18}$, os discos com degeneração superior ao Tipo II são considerados doentes. Nosso estudo apresentou uma frequência de $85,8 \%$ dos discos doentes. Leite et al..$^{19}$ observaram uma frequência menor, provavelmente pela inclusão dos níveis L1-L2 e L2-L3 e pela menor média de idade dos pacientes avaliados.

Dados a partir de análises anatomopatológicas revelaram que o sexo não tem efeito significativo sobre a degeneração do disco lombar. ${ }^{16}$ No entanto, estudos anteriores mostraram que as mulheres alcançam o mesmo nível de alterações degenerativas discais 10 anos mais tarde em relação aos homens, este dado pode está relacionado a maior frequência de atividades físicas pesadas pelos homens, esta mesma relação foi encontrada entre trabalhadores com diferentes atividades em relação ao esforço físico. ${ }^{20}$ Nosso estudo mostrou que em relação à maioria dos níveis e ao total de níveis analisados não houve diferença entre os sexos em relação ao grau de degeneração discal.

A avaliação do grau de tropismo facetário mostrou que o grau de assimetria foi bastante semelhante entre os níveis analisados. Kalichman et al. ${ }^{7}$ observaram que não existe diferenças significativas na distribuição do tropismo de acordo do o sexo. Nossa análise mostrou que os homens apresentam graus de assimetrias estatisticamente maiores no nível L5-S1, o que poderia explicar a maior frequência de certas patologias ser mais frequentes em homens no nível em questão, como a espondilolistese. Sabe-se que a assimetrias aumentam a força rotacional de um lado da coluna vertebral, ocorrendo espondilólise no lado onde a faceta é mais coronalmente orientada. ${ }^{21}$

Estudo anterior analisando os mesmos níveis não observou associações de tropismo moderado e grave com a idade. ${ }^{7}$ Diferentemente, nosso estudo observou o aumento do tropismo com o aumento da idade na maioria dos níveis e em relação o total de níveis analisados. 
O tropismo articular pode levar a instabilidade lombar manifestando-se com aumento da rotação local e a consequente tensão adicional sobre o disco intervertebral. A literatura é concordante que o tropismo facetário não está associado à protrusão discal lombar. ${ }^{22-24}$ Nosso estudo mostrou quanto maior o grau de tropismo maior o grau de degeneração discal em concordância com Noren et $a^{1}{ }^{10}$ No entanto, ainda é um assunto controverso, não sendo encontrada a mesma relação por Cassidy et al. ${ }^{8}$ e Vanharanta et al. ${ }^{9}$

Em concordância com Kalichman et al. ${ }^{7}$ não houve diferença significativa nos valores médios da orientação das facetas em relação a idade e sexo.

\section{CONCLUSÃO}

A maioria dos discos intervertebrais analisados de pacientes com dor lombar crônica encontra-se degenerados e grau de degeneração aumenta com a idade.

O grau de tropismo facetário aumenta com a idade e se relaciona com o grau de degeneração discal.

Todos os autores declaram não haver nenhum potencial conflito de interesses referente a este artigo.

\section{REFERÊNCIAS}

1. Henrotin $Y E$, Cedraschi C, Duplan B, Bazin T, Duquesnoy B. Information and low back pain management: a systematic review. Spine (Phila Pa 1976). 2006;31(11):E326-34.

2. Coppes MH, Marani E, Thomeer RT, Groen GJ. Innervation of "painful" lumbar discs. Spine (Phila Pa 1976). 1997;22(20):2342-9.

3. Kuslich SD, Ulstrom CL, Michael CJ. The tissue origin of low back pain and sciatica: a report of pain response to tissue stimulation during operations on the lumbar spine using local anesthesia. Orthop Clin North Am. 1991;22(2):181-7.

4. Manchikanti L, Singh V, Pampati V, Damron KS, Barnhill RC, Beyer C, et al. Evaluation of the relative contributions of various structures in chronic low back pain. Pain Physician. 2001;4(4):308-16.

5. César AEM, Yonezaki AMY, Ueno FH, Filho ESVF, Rodrigues LMRR. Reprodutibilidade intra e interobservadores da classificação de hipersinal facetário lombar e correlação com a degeneração discal para ressonância magnética. Coluna/Columna. 2011;10(3):179-82.

6. Silva CIS, D'Ippolito G, Rocha AJ. Doença degenerativa da coluna vertebral. In: Fernades JL. Coluna vertebral. Rio de Janeiro: Elsevier; 2011. p.153-98.

7. Kalichman L, Suri P, Guermazi A, Li L, Hunter DJ. Facet orientation and tropism: associations with facet joint osteoarthritis and degeneratives. Spine (Phila Pa 1976). 2009;34(16):E579-85

8. Cassidy JD, Loback D, Yong-Hing K, Tchang S. Lumbar facet joint asymmetry. Intervertebral disc herniation. Spine (Phila Pa 1976). 1992;17(5):570-4

9. Vanharanta H, FloydT, Ohnmeiss DD, Hochschuler SH, Guyer RD. The relationship of facet tropism to degenerative disc disease. Spine (Phila Pa 1976). 1993;18(8):1000-5.

10. Noren R, Trafimow J, Andersson GB, Huckman MS. The role of facet joint tropism and facet angle in disc degeneration. Spine (Phila Pa 1976). 1991;16(5):530-2.

11. Wang J, Yang X. Age-related changes in the orientation of lumbar facet joints. Spine (Phila Pa 1976). 2009;34(17):E596-8

12. Cyron BM, Hutton WC. Articular tropism and stability of the lumbar spine. Spine (Phila Pa 1976). 1980;5(2):168-72.
13. Pfirrmann CW, Metzdorf A, Zanetti M, Hodler J, Boos N. Magnetic resonance classification of lumbar intervertebral disc degeneration. Spine (Phila Pa 1976). 2001;26(17):1873-8.

14. Biyani A, Haman SP, Andersson GBJ. Lumbar disc desease. In: Rothman-Simeone. The spine. 5th ed. Philadelphia: Saunders Elsevier; 2006. p. 930-944.

15. Lam K, Anbar A, O'Brien A. The Correlation of the severity of lumbar disc degeneration with discogênica low back pain - A study utilizing a validated a classification with awake provocative discography. J Bone Joint Surg Br. 2010;92:566.

16. Siemionow K, An H, Masuda K, Andersson G, Cs-Szabo G. The effects of age, sex, ethnicity, and spinal level on the rate of intervertebral disc degeneration: a review of 1712 intervertebral discs. Spine (Phila Pa 1976). 2011;36(17):1333-9

17. Miller JA, Schmatz C, Schultz AB. Lumbar disc degeneration: correlation with age, sex and spine level in 600 autopsy specimens. Spine (Phila Pa 1976). 1988;13(2):173-8.

18. Zuo J, Saadat E, Romero A, Loo K, Li X, LinkTM, et al. Assessment of intervertebral disc degeneration with magnetic resonance single-voxel spectroscopy. Magn Reson Med. 2009;62(5):1140-6

19. Leite MS, Luciano RP, Martins ED.Correlação entre as classificações de Pfirrmann e Modic na degeneração do disco intervertebral lombar. Coluna/Columna. 2010;9(4):401-6.

20. Battié MC, Videman T, Parent E. Lumbar disc degeneration: epidemiology and genetic influences. Spine (Phila Pa 1976). 2004:29(23):2679-90

21. Rankine JJ, Dickson RA. Unilateral spondylolysis and the presence of facet joint tropism. Spine (Phila Pa 1976). 2010;35(21):E1111-4.

22. Lee DY, Ahn Y, Lee SH. The influence of facet tropism on herniation of the lumbar disc in adolescents and adults. J Bone Joint Surg Br. 2006;88(4):520-3.

23. Yu H, Hou S, Wu W, Zhou B. The relationship of facet orientation to intervertebral disc protrusion and lateral recess stenosis in lower lumbar spine. Zhonghua Wai Ke Za Zhi. 1998:36(3):176-8.

24. Dai LY. Orientation and tropism of lumbar facet joints in degenerative spondylolisthesis. Int Orthop. 2001;25(1):40-2 